\title{
Calculating the Electron Paramagnetic Resonance Parameters of Exchange Coupled Transition Metal Complexes Using Broken Symmetry Density Functional Theory. Application to a $\mathrm{Mn}^{\mathrm{III}} / \mathrm{Mn}^{\mathrm{IV}}$ Model Compound.
}

Sebastian Sinnecker, Frank Neese, Louis Noodleman and Wolfgang Lubitz

\section{Supporting Information}

\section{Contents}

- Cartesian coordinates of the $\left[\mathrm{Mn}^{\mathrm{III}} \mathrm{Mn}^{\mathrm{IV}}(\mu-\mathrm{O})_{2}(\mu-\mathrm{OAc}) \mathrm{DTNE}\right]^{2+}$ complex from geometry optimization

- Charges and net spin densities of the bs state

- Heisenberg exchange coupling constants according to Yamaguchi's equation

- $g$-tensor site values

- Alternative approach for the calculation of $g$-tensor site values and $g$-tensors

- ${ }^{55} \mathrm{Mn}$ hyperfine coupling constants and nuclear quadrupole tensors from Hyper2000 


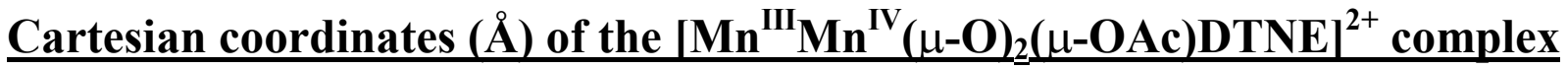

from geometry optimization

Level of calculation: ADF program, BP functional. Basis sets IV (Mn), III (other elements). 1s frozen core for $\mathrm{C}, \mathrm{N}$ and $\mathrm{O}(\mathrm{ADF}-1)$. Best agreement between theory and experiment.

\begin{tabular}{|c|c|c|c|c|c|c|c|}
\hline Mn & 2.823457 & 1.655222 & 3.889289 & $\mathrm{H}$ & 0.724802 & 4.532025 & 5.465791 \\
\hline Mn & 1.998963 & -0.627812 & 3.100151 & $\mathrm{H}$ & 4.201476 & 2.864724 & 6.296492 \\
\hline $\mathrm{O}$ & 4.109948 & 0.500291 & 5.181562 & $\mathrm{H}$ & 3.639498 & 4.554854 & 6.417859 \\
\hline $\mathrm{O}$ & 3.228921 & -1.437477 & 4.376753 & $\mathrm{H}$ & 4.252113 & 5.010144 & 4.091741 \\
\hline $\mathrm{O}$ & 1.362120 & 0.628440 & 4.221113 & $\mathrm{H}$ & 5.623512 & 4.172933 & 4.815186 \\
\hline $\mathrm{O}$ & 3.179587 & 0.570004 & 2.467326 & $\mathrm{H}$ & 4.316949 & 2.666618 & 1.404208 \\
\hline $\mathrm{N}$ & 1.753064 & 3.111506 & 2.522144 & $\mathrm{H}$ & 4.916531 & 4.296936 & 1.815146 \\
\hline $\mathrm{N}$ & 2.392337 & 3.188672 & 5.299134 & $\mathrm{H}$ & 2.693299 & 5.016030 & 2.557578 \\
\hline $\mathrm{N}$ & 4.435081 & 2.992433 & 3.460841 & $\mathrm{H}$ & 2.552897 & 4.340163 & 0.944931 \\
\hline $\mathrm{N}$ & 0.549610 & -0.131739 & 1.642526 & $\mathrm{H}$ & -0.671495 & -1.822221 & 1.186917 \\
\hline $\mathrm{N}$ & 0.597574 & -2.099268 & 3.648312 & $\mathrm{H}$ & -1.592418 & -0.406230 & 1.673272 \\
\hline $\mathrm{N}$ & 2.506784 & -2.118679 & 1.700633 & $\mathrm{H}$ & -0.869660 & -0.664857 & 4.033539 \\
\hline $\mathrm{C}$ & 4.025381 & -0.756714 & 5.150541 & $\mathrm{H}$ & -1.547447 & -2.211676 & 3.449205 \\
\hline $\mathrm{C}$ & 4.897068 & -1.569776 & 6.063110 & $\mathrm{H}$ & 0.086669 & -3.576909 & 2.215369 \\
\hline $\mathrm{C}$ & 1.081904 & 2.443635 & 1.367495 & $\mathrm{H}$ & 0.759249 & -4.243730 & 3.702054 \\
\hline $\mathrm{C}$ & 0.085472 & 1.308855 & 1.641833 & $\mathrm{H}$ & 3.017437 & -3.536358 & 3.144090 \\
\hline $\mathrm{C}$ & 0.794630 & 3.767707 & 3.450199 & $\mathrm{H}$ & 2.359166 & -4.271455 & 1.655239 \\
\hline $\mathrm{C}$ & 1.489811 & 4.241923 & 4.729341 & $\mathrm{H}$ & 0.978498 & -2.671770 & 0.344326 \\
\hline $\mathrm{C}$ & 3.729704 & 3.688131 & 5.740965 & $\mathrm{H}$ & 2.436337 & -2.084694 & -0.448153 \\
\hline $\mathrm{C}$ & 4.570571 & 4.048927 & 4.518938 & $\mathrm{H}$ & 2.026505 & 0.203905 & 0.189944 \\
\hline $\mathrm{C}$ & 4.187186 & 3.515819 & 2.090404 & $\mathrm{H}$ & 0.491261 & -0.410136 & -0.495766 \\
\hline $\mathrm{C}$ & 2.769942 & 4.076932 & 1.993630 & $\mathrm{H}$ & 0.681044 & -2.205674 & 4.669124 \\
\hline $\mathrm{C}$ & -0.680874 & -0.982852 & 1.893578 & $\mathrm{H}$ & 3.518812 & -2.017181 & 1.538482 \\
\hline $\mathrm{C}$ & -0.719957 & -1.492234 & 3.325332 & $\mathrm{H}$ & 1.927768 & 2.747929 & 6.105534 \\
\hline $\mathrm{C}$ & 0.858963 & -3.418066 & 2.981152 & $\mathrm{H}$ & 5.287053 & 2.414328 & 3.444231 \\
\hline $\mathrm{C}$ & 2.254544 & -3.430962 & 2.361690 & & & & \\
\hline $\mathrm{C}$ & 1.771565 & -1.915528 & 0.412876 & & & & \\
\hline $\mathrm{C}$ & 1.201722 & -0.502378 & 0.345259 & & & & \\
\hline $\mathrm{H}$ & 4.711018 & -2.643632 & 5.956948 & & & & \\
\hline $\mathrm{H}$ & 4.716351 & -1.262749 & 7.103142 & & & & \\
\hline $\mathrm{H}$ & 5.952091 & -1.354094 & 5.840559 & & & & \\
\hline $\mathrm{H}$ & 1.881088 & 2.100552 & 0.698756 & & & & \\
\hline $\mathrm{H}$ & 0.514048 & 3.216298 & 0.814238 & & & & \\
\hline $\mathrm{H}$ & -0.421762 & 1.458062 & 2.603018 & & & & \\
\hline $\mathrm{H}$ & -0.682025 & 1.378626 & 0.850781 & & & & \\
\hline $\mathrm{H}$ & 0.034882 & 3.022582 & 3.721308 & & & & \\
\hline $\mathrm{H}$ & 0.279868 & 4.623921 & 2.973857 & & & & \\
\hline $\mathrm{H}$ & 2.089614 & 5.141780 & 4.539518 & & & & \\
\hline
\end{tabular}




\section{Charges and net spin densities of the bs state}

Mulliken population analysis

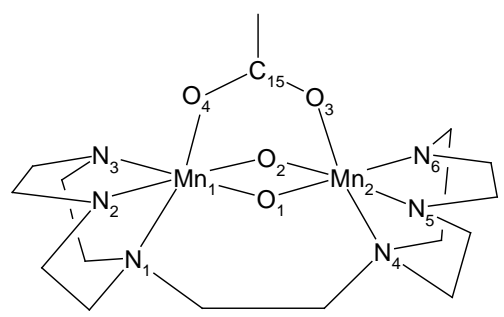

\begin{tabular}{|c|c|c|c|c|c|c|c|c|c|c|}
\hline & \multicolumn{2}{|c}{ ORCA-1 } & \multicolumn{2}{c|}{ ORCA-2 } & \multicolumn{2}{c|}{ ADF-1 } & \multicolumn{2}{c|}{ ADF-2 } & \multicolumn{3}{c|}{ ADF-3 } \\
\hline & charge & net spin & charge & net spin & charge & net spin & charge & net spin & charge & net spin \\
\hline $\mathrm{Mn}_{1}$ & +0.689 & +3.472 & +0.855 & +3.866 & +1.173 & +3.426 & +1.072 & +3.461 & +1.050 & +3.438 \\
\hline $\mathrm{Mn}_{2}$ & +0.642 & -2.367 & +0.851 & -2.731 & +1.258 & -2.313 & +1.132 & -2.332 & +1.031 & -2.319 \\
\hline $\mathrm{O}_{1}$ & -0.467 & -0.114 & -0.559 & -0.151 & -0.822 & -0.113 & -0.800 & -0.123 & -0.691 & -0.116 \\
\hline $\mathrm{O}_{2}$ & -0.468 & -0.123 & -0.560 & -0.162 & -0.817 & -0.129 & -0.794 & -0.139 & -0.685 & -0.132 \\
\hline $\mathrm{O}_{4}$ & -0.349 & +0.007 & -0.402 & +0.028 & -0.795 & +0.010 & -0.791 & +0.011 & -0.650 & +0.010 \\
\hline $\mathrm{C}_{15}$ & +0.312 & -0.003 & +0.362 & -0.004 & +1.054 & -0.002 & +1.066 & -0.002 & +0.806 & -0.003 \\
\hline $\mathrm{O}_{3}$ & -0.369 & +0.035 & -0.435 & +0.044 & -0.791 & +0.030 & -0.781 & +0.033 & -0.657 & +0.031 \\
\hline $\mathrm{N}_{1}$ & -0.449 & +0.028 & -0.510 & +0.052 & -0.616 & +0.032 & -0.611 & +0.033 & -0.530 & +0.031 \\
\hline $\mathrm{N}_{2}$ & -0.344 & -0.029 & -0.386 & -0.031 & -0.625 & -0.022 & -0.618 & -0.021 & -0.472 & -0.022 \\
\hline $\mathrm{N}_{3}$ & -0.341 & -0.028 & -0.383 & -0.029 & -0.617 & -0.023 & -0.611 & -0.023 & -0.465 & -0.023 \\
\hline $\mathrm{N}_{4}$ & -0.446 & +0.038 & -0.501 & +0.042 & -0.610 & +0.031 & -0.599 & +0.031 & -0.518 & +0.031 \\
\hline $\mathrm{N}_{5}$ & -0.321 & +0.037 & -0.358 & +0.029 & -0.604 & +0.031 & -0.595 & +0.030 & -0.449 & +0.031 \\
\hline $\mathrm{N}_{6}$ & -0.325 & +0.036 & -0.362 & +0.029 & -0.606 & +0.031 & -0.596 & +0.029 & -0.451 & +0.030 \\
\hline
\end{tabular}

Table S1

\section{Heisenberg exchange coupling constants $\left(\mathrm{cm}^{-1}\right)$ according to Yamaguchi's equation $^{1,2}$}

Spin expectation values are available only in ORCA, but not in ADF.

\begin{tabular}{|c|c|c|}
\hline & $\begin{array}{c}\text { BP } \\
\text { ORCA-1 }\end{array}$ & $\begin{array}{c}\text { B3LYP } \\
\text { ORCA-2 }\end{array}$ \\
\hline bs//hs-opt & -230 & -119 \\
\hline bs-opt/hs-opt & -284 & -126 \\
\hline
\end{tabular}

$$
J=-\frac{E_{H S}-E_{B S}}{\left\langle S_{H S}^{2}\right\rangle-\left\langle S_{B S}^{2}\right\rangle}
$$

Table S2

\footnotetext{
${ }^{1}$ Soda, T.; Kitagawa, Y.; Onishi, T.; Takano, Y.; Shigeta, Y.; Nagao, H.; Yoshioka, Y.; Yamaguchi, K. Chem. Phys. Lett. 2000, 319, 223-230.

${ }^{2}$ Yamaguchi, K.; Takahara, Y.; Fueno, T. In Applied Quantum Chemistry; Smith, V. H., Schaefer, F., Morokuma, K., Eds. Reidel: Dordrecht, 1986; p 155.
} 


\section{g-tensor site values}

\begin{tabular}{|c|c|c|c|c|c|c|c|c|}
\hline & \multicolumn{2}{|c|}{ ORCA-1 } & \multicolumn{2}{c|}{ ORCA-2 } & \multicolumn{2}{c|}{ ORCA-3 } & \multicolumn{2}{c|}{ exptl. $^{3}$} \\
\hline site & $M n^{I I}$ & $M n^{I V}$ & $M n^{I I I}$ & $M n^{I V}$ & $M n^{I I I}$ & $M n^{I V}$ & $M n^{I I I}$ & $M n^{I V}$ \\
\hline$g_{x}$ & 1.995 & 2.003 & 2.001 & 1.998 & 2.001 & 1.999 & 1.9945 & 1.9879 \\
\hline$g_{y}$ & 1.998 & 2.002 & 2.000 & 1.999 & 2.000 & 1.999 & 1.9921 & 1.9870 \\
\hline$g_{z}$ & 1.988 & 2.000 & 1.992 & 2.001 & 1.992 & 2.001 & 1.9872 & 1.9907 \\
\hline
\end{tabular}

Table S3

\section{Alternative approach for the calculation of $g$-tensor site values and $g$-tensors}

In this work, the $g$-tensor of the bs state is calculated as a sum of one-electron excitations that can be assigned to site 1 or site 2 excitations, depending on the location of the respective molecular orbitals. The separate summation of these contributions is another way for the calculation of the $g$-tensor site values, given here. Subsequently, spin projection was applied for the calculation of the molecular $g$-tensor (Table S4).

Some of the excitations could not be unambiguously classified and were taken into account for both sites.

\begin{tabular}{|c|c|c|c|c|}
\hline & BP & B3LYP & B3LYP//BP & exptl. \\
\hline$g_{x}$ & 1.999 & 1.995 & 1.996 & 2.0013 \\
\hline$g_{y}$ & 1.996 & 1.993 & 1.992 & 1.9974 \\
\hline$g_{z}$ & 1.985 & 1.983 & 1.985 & 1.9838 \\
\hline$g_{\text {iso }}$ & 1.993 & 1.990 & 1.991 & 1.9942 \\
\hline
\end{tabular}

\section{Table S4}

\footnotetext{
${ }^{3}$ Schäfer, K. O. Exchange Coupled Manganese Complexes: Model Systems for the Active Centres of Redoxproteins Investigated with EPR Techniques; Dissertation; Technische Universität Berlin, Germany, 2002.
} 


\section{${ }^{55}$ Mn hyperfine coupling constants and nuclear quadrupole tensors from Hyper2000}

Hyper2000 (T. Liu, L. Noodleman and D. A. Case) is a program developed for the calculation of Mössbauer and EPR parameters from the TAPE 21 and TAPE 10 data files generated by ADF.

Comparison of the calculated ${ }^{55} \mathrm{Mn}$ hyperfine and quadrupole data $(\mathrm{MHz})$ obtained directly from ADF and computed with HYPER2000 (ADF1: 1s frozen core for C, N and O. ADF-3: no frozen core).

\begin{tabular}{|l|c|c|c|c|c|c|}
\hline & & $\begin{array}{c}\text { HYPER2000 } \\
\text { ADF-1 }\end{array}$ & ADF-1 & $\begin{array}{c}\text { HYPER2000 } \\
\text { ADF-3 }\end{array}$ & ADF-3 & exptl. \\
\hline $\mathrm{Mn}^{\mathrm{III}}$ & $A_{\text {iso }}$ & -163 & -164 & -163 & -164 & -389 \\
\hline & $A_{x}^{\prime}$ & -67 & -61 & -67 & -61 & -17 \\
\hline & $A_{y}^{\prime}$ & -14 & -25 & -14 & -25 & -81 \\
\hline & $A_{z}^{\prime}$ & +82 & +85 & +82 & +86 & +92 \\
\hline & $P_{x}$ & +2.66 & +1.82 & +2.66 & +1.89 & \\
\hline & $P_{y}$ & -0.18 & -0.28 & -0.17 & -0.27 & \\
\hline \multirow{3}{*}{$\mathrm{Mn}^{\mathrm{IV}}$} & $P_{z}$ & -2.49 & -1.55 & -2.49 & -1.11 & \\
\hline & $A_{i s o}$ & +83 & +84 & +83 & +84 & +207 \\
\hline & $A_{x}^{\prime}$ & -3 & -8 & -3 & -8 & +14 \\
\hline & $A_{y}^{\prime}$ & +19 & +26 & +19 & +26 & -14 \\
\hline & $A_{z}^{\prime}$ & -16 & -18 & -16 & -18 & +6 \\
\hline & $P_{x}$ & -0.60 & -1.38 & -0.64 & -1.37 & \\
\hline & $P_{y}$ & -2.32 & -2.16 & -2.29 & -2.10 & \\
\hline & $P_{z}$ & +2.92 & +3.54 & +2.93 & +3.47 & \\
\hline
\end{tabular}

\section{Table S5}

